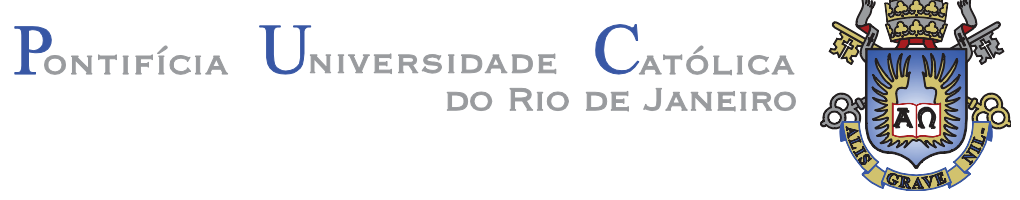

José Mauricio Nava Auza

\title{
Análise de Desempenho de Algoritmos de Eficiência Energética em RSSF
}

Dissertação de Mestrado

Dissertação apresentada ao Programa de Pós-graduação em Engenharia Elétrica do Departamento de Engenharia Elétrica do Centro Técnico Científico da PUC-Rio como requisito parcial para obtenção do grau de Mestre em Engenharia Elétrica

Orientador: Prof. José Roberto Boisson de Marca

Rio de Janeiro

Setembro de 2013 
Pontifícia Universidade Católica $_{\text {a }}$

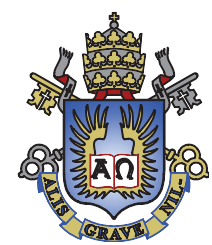

José Mauricio Nava Auza

\section{Análise de Desempenho de Algoritmos de Eficiência Energética em RSSF}

Dissertação apresentada ao Programa de Pósgraduação em Engenharia Elétrica do Departamento de Engenharia Elétrica do Centro Técnico Científico da PUC-Rio como requisito parcial para obtenção do grau de Mestre em Engenharia Elétrica. Aprovada pela Comissão Examinadora abaixo assinada.

Prof. José Roberto Boisson de Marca Orientador

Centro de Estudos em Telecomunicações -PUC-Rio

Prof. Guilherme Dutra Gonzaga Jaime UFRJ

Prof. Luiz Alencar Reis da Silva Mello Centro de Estudos em Telecomunicações -PUC-Rio

Prof. José Eugênio Leal Coordenador Setorial do Centro Técnico Científico- PUC-Rio 
Todos os direitos reservados. É proibida a reprodução total ou parcial do trabalho sem autorização da universidade, do autor e do orientador.

José Mauricio Nava Auza

Engenheiro em Telecomunicações graduado pela Universidade Católica Boliviana, La Paz-Bolivia em 2010

Ficha Catalográfica

Análise de Desempenho de Algoritmos de Eficiência Energética em RSSF / José Mauricio Nava Auza; orientador: José Roberto Boisson de Marca.-2013.

V., 94 f: il. ; $30 \mathrm{~cm}$

Dissertação (mestrado) - Pontifícia Universidade Católica do Rio de Janeiro, Departamento de Engenharia Elétrica.

Incluí referências bibliográficas.

1. Engenharia Elétrica-Tese. 2. RSSF. 3. Teoria dos grafos. 4. Potência de transmissão. 5. Algoritmos de eficiência energética. 6. LQI. 7. RSSI. I. Boisson de Marca, José Roberto. II. Pontifícia Universidade Católica do Rio de Janeiro. Departamento de Engenharia Elétrica. III. Título. 
A los 7 pilares que supieron sostenerme a lo largo de esta etapa, José, Daphne, Valeria, Jaime, Sofia, Ignacio y Victoria. 


\section{Agradecimentos}

Sem duvida alguma, no momento de escrever esta folha eu sinto uma enorme satisfação de ter concluído mais uma etapa na minha vida e quero aproveitar para agradecer a todos aqueles que me ajudaram a cumprir este objetivo, já que sem o apoio e a ajuda deles não teria conseguido chegar a este momento.

Quero começar agradecendo a Deus que me abençoou com esta oportunidade e me deu saúde e fortaleza para afrontar este desafio fora do meu país.

São muitas as pessoas que contribuíram nesta dissertação direta e indiretamente. Primeiramente quero agradecer aos meus tios Jaime e Sofia que me receberam na casa deles, na qual me senti muito cômodo ao longo deste período, pois fizeram me sentir em casa e em todo momento me apoiaram e acompanharam nas frustrações e alegrias que este projeto me trouxe. Quero agradecer aos meus pais, José e Daphne, eles que me ensinaram aquelas coisas das quais não se falam nos livros, eles são o meu maior exemplo de vida, obrigado por terem feito de mim um guerreiro. A minha irmã Valeria, por sempre se preocupar comigo; obrigado por fazer eu me sentir importante. A distancia não conseguiu nos separar e sempre senti vocês muito perto, obrigado por sempre ter a palavra exata. Aos meus primos Ignacio e Victoria, obrigado por estarem perto de mim. A minha avó, Tuquita, que sempre acredito em mim.

A todos os companheiros do CETUC pelo apoio ao longo deste tempo, especialmente a Cesar, Diego e Juliana. Ao Professor José Roberto Boisson de Marca por aceitar me orientar nesta dissertação, pela compreensão e por todos os conhecimentos compartilhados.

Não posso me esquecer daquelas pessoas que me apoiaram em momentos difíceis; Adriano Branco, muito obrigado por toda sua ajuda neste projeto, onde sem você nada disto teria sido possível. Aprendi muito contigo amigo. A Jennifer que me fez acreditar em mim quando nem eu mesmo acreditava; obrigado por todo o apoio e alegria que você trouxe para a minha vida. A Alberth, sem duvida alguma um dos melhores amigos que este processo me deixou, obrigado por todas as conversas e a ajuda dada.

Por ultimo quero agradecer a PUC-Rio e a agencia CAPES pelo apoio financeiro promovido e por acreditar em meu trabalho. 


\section{Resumo}

Nava Auza, José Mauricio; Boisson de Marca, José Roberto. Análise de Desempenho de Algoritmos de Eficiência Energética em RSSF. Rio de Janeiro, 2013. 94p. Dissertação de Mestrado - Departamento de Engenharia Elétrica, Pontifícia Universidade Católica do Rio de Janeiro.

As redes de sensores sem fio se constituem numa área que outorga grandes oportunidades para a oferta de uma série de aplicações inovadoras e com baixo custo. Os dispositivos destas redes são bastantes pequenos e sua fonte de alimentação são baterias. O tempo de vida destas é limitado, limitando assim o tempo da vida dos sensores e da rede como um todo. Por esta razão nos últimos anos o tema de eficiência energética tem atraído grande interesse de pesquisadores. O aumento do custo da energia e do consumo global da energia pelo setor de ICT (Information and Communications Technologies) têm crescido vertiginosamente devido ao aumento continuo do número de clientes e da demanda por aplicações de maior complexidade. Por tudo isso têm sido desenvolvidos distintos métodos e técnicas para economizar energia nas RSSF. Neste trabalho se implementam dois algoritmos que levam em conta critérios para economizar os custos de energia da rede e através de experimentos de simulação se avalia os mesmos. Nos resultados pode se observar as vantagens de trabalhar com sistemas que visam a eficiência energética.

\section{Palavras-chave}

RSSF; teoria dos grafos; potência de transmissão; algoritmos de eficiência energética; LQI; RSSI. 


\section{Abstract}

Nava Auza, José Mauricio; Boisson de Marca, José Roberto(Advisor). Performance of Energy Efficient algorithms in WSN. Rio de Janeiro, 2013. 94p. MSc. Dissertation - Departamento de Engenharia Elétrica, Pontifícia Universidade Católica do Rio de Janeiro.

The WSNs (Wireless Sensor Networks) belong to an area that gives rise to great opportunities to spread innovative and low cost applications. These kinds of networks are composed of tiny devices with limited energy. The main source of power supply for WSNs are batteries, which are limited in cycle life, thus limiting the sensors lifetime and the network as a whole. Due to that fact, the energy efficiency network is becoming the main concern to be addressed by researchers. Rising energy prices and global energy consumption by the ICT (Information and Communications Technologies) sector have grown dramatically due to the continuous increase in customer number and the demand for more complex applications. For the reasons outlined above, different energy-saving techniques for WSNs have been developed. Two energy-saving algorithms for WSNs were implemented in this thesis, and they were tested by experimental evaluation using simulation. The results obtained from the simulations showed the advantages of working with systems aiming at energy efficiency.

\section{Keywords}

WSN; Graph Theory; Transmission Power; Energy efficient algorithms; LQI; RSSI. 


\section{Sumário}

1 Introdução 15

1.1. Motivação e objetivo 15

1.2. Trabalhos Relacionados 17

1.3. Contribuições 19

1.4. Estrutura da dissertação 20

2 Aspectos Teóricos $\quad 21$

2.1. Redes de sensores sem fio $\quad 21$

2.2 Teoria dos grafos 23

2.2.1 Grafo Planar 26

$\begin{array}{ll}\text { 2.2.2 Grafo do disco unitário } & 27\end{array}$

2.3. Controle de topologia 28

2.3.1 Relative neighboorhood graph $\quad 30$

2.3.2 Minimum Spanning Tree $\quad 31$

2.4 Padrões e Tecnologias $\quad 32$

2.4.1 IEEE 802.15 33

2.5 MicaZ 36

$\begin{array}{lll}2.5 .1 & \mathrm{CC} 2420 & 37\end{array}$

3 Ferramentas de Simulação e Algoritmos $\quad 41$

3.1 Características e comparações das ferramentas para simulação 41

3.2 Algoritmos Propostos $\quad 45$

3.2.1 DTNBOR 46

3.2.2 DTRNG $\quad 54$

$\begin{array}{ll}\text { 3.2.3 Protocolo de encaminhamento } & 60\end{array}$

4 Analise de Resultados $\quad 64$

4.1 Esquema de Simulação $\quad 64$

$\begin{array}{ll}\text { 4.1.2 Métricas } & 67\end{array}$ 
$\begin{array}{ll}\text { 4.2 Cenário } A & 68\end{array}$

4.3 Cenário $B \quad 69$

$\begin{array}{ll}4.4 \text { Cenário } \mathrm{C} & 76\end{array}$

4.5 Cenário D $\quad 79$

5 Conclusões $\quad 86$

6 Referências Bibliográficas $\quad 88$

$\begin{array}{ll}\text { Apêndice A } & 92\end{array}$ 


\section{Lista de figuras}

Figura 2.1: Arquitetura de uma rede de sensores 22

Figura 2.2: Estrutura de um nó sensor 23

Figura 2.3: Nomenclatura dos grafos $\quad 24$

Figura 2.4: Tipos de grafos $\quad 24$

Figura 2.5: Classificação dos grafos $\quad 25$

Figura 2.6: Exemplos de grafo planar 26

Figura 2.7: Grafo planar de um cubo $\quad 27$

Figura 2.8: Comportamento do grafo do disco unitário 27

Figura 2.9: Exemplos de estruturas geométricas 29

Figura 2.10: O RNG de um triângulo. 30

Figura 2.11: Conceito Lua 30

Figura 2.12: Exemplos de formação de ciclos 31

Figura 2.13: Exemplo de mais de um MST num grafo 32

Figura 2.14: Família de padrões do IEEE 802.11

Figura 2.15: Família de padrões 802.15 34

Figura 2.16: Topologias compatíveis com Zigbee 35

Figura 2.17: Aplicações do Zigbee 36

Figura 2.18: Aspecto físico do Micaz $\quad 37$

Figura 2.19: Descrição de funcionamento de um Micaz 37

Figura 2.20: Quadro do 802.15.4 40

Figura 2.21: Uso dos dois bytes do FCS 40

Figura 3.1: Diagrama em blocos da metodologia de simulação 45

Figura 3.2: Diagrama de fluxo do algoritmo DTNBOR 47

Figura 3.3: Interpolação do Avrora para níveis de potência. Fuente: AvroraZ 48

Figura 3.4: Trama da Mensagem Hello e Resposta 49

Figura 3.5: Descrição dos temporizadores $T_{\text {Presposta }}$ e $T_{\text {resposta }}$

Figura 3.6: Diagrama de fluxo do algoritmo DTNBOR adequado a TinyOS 53

Figura 3.7: Exemplo da equação 5

Figura 3.8: Diagrama de fluxo do algoritmo DTRNG 56

Figura 3.9: Montagem da tabela de informações locais 57 
Figura 3.10: Exemplo de escolha de potências para condição do DTRNG 58

Figura 3.11: Diagrama de fluxo do algoritmo DTRNG adequado a TinyOS 59

Figura 3.12: Descrição da fila implementada 61

Figura 3.13: Diagrama do protocolo de encaminhamento 63

Figura 4.1: Esquema de simulação 64

Figura 4.2: Esquema para obtenção de resultados com potências máximas 65

Figura 4.3: Esquema de simulação para sistema com potências variáveis $\quad 66$

Figura 4.4 Esquema de simulação para sistema com potências variáveis $\quad 67$

Figura 4.5: Grafo de comunicação da topologia A 68

Figura 4.6: Grafo de comunicação do protocolo de coleta de dados

para a Topologia A $\quad 68$

Figura 4.7: Grafo de comunicação do protocolo de coleta de dados

com as potências da tabela criada por TinyDTRNG para a Topologia B 69

Figura 4.9: Grafo de comunicação do protocolo de coleta de dados

para a Topologia B $\quad 70$

Figura 4.10: Grafo de comunicação do protocolo de coleta de dados com as potências da tabela criada por TinyDTRNG para a Topologia B 71

Figura 4.11: Coleta de dados com potências máximas para a Topologia B 72

Figura 4.12: Coleta de dados com potências variáveis de TinyDTNBOR para a Topologia B

Figura 4.13: Coleta de dados com potências variáveis de TinyDTRNG

para a Topologia $B$

Figura 4.14: Economia de energia na transmissão de TinyDTNBOR

e TinyDTRNG respeito a Potências Máximas

Figura 4.15: Extrapolação dos resultados obtidos para um tempo igual a uma semana de funcionamento para a topologia B 75

Figura 4.16: Analise do comportamento da aplicação com TinyDTRNG 76

Figura 4.17: Grafo de comunicação da topologia C 77

Figura 4.18: Grafo de comunicação do protocolo de coleta de dados

para a Topologia C

Figura 4.19: Coleta de dados com potências máximas e variáveis de para a Topologia $C$

Figura 4.20: Economia de energia na transmissão de DTNBOR com respeito a Potências Máximas

Figura 4.21: Extrapolação dos resultados obtidos para um tempo igual a uma semana de funcionamento para a topologia $\mathrm{C}$ 
Figura 4.22: Curva de PRR para o nível 31 de potência 80

Figura 4.23: RSSI para o nível de Potência $31 \quad 80$

Figura 4.24: LQI para o nível de Potência 31

Figura 4.25: Comportamento do LQI com respeito ao BER 81

Figura 4.26: Comparação de métricas respeito a distância para

o nível de potência 27

82

Figura 4.27: Comparação de métricas em função da distância para

o nível de potência 19

Figura 4.28: Grafo de comunicação da topologia D 83

Na Figura 4.29 é apresentada a topologia com as mudanças causadas

pelo protocolo de encaminhamento. 83

Figura 4.29: Grafo de comunicação do protocolo de coleta de dados

para a Topologia D 83

Figura 4.30: Coleta de dados com potências máximas e variáveis de para a Topologia D

Figura 4.31: Extrapolação dos resultados obtidos para um tempo igual a uma semana de funcionamento para a topologia $C$

Figura A.1: Comparação de métricas em função da distância para

o nível de potência 31

Figura A.2: Comparação de métricas em função da distância para

o nível de potência 23

Figura A.3: Comparação de métricas em função da distância para

o nível de potência 15

Figura A.4: Comparação de métricas em função da distância para o nível de potência 11

Figura A.5: Comparação de métricas em função da distância para o nível de potência 7

Figura A.6: Comparação de métricas em função da distância para o nível de potência 3 


\section{Lista de tabelas}

Tabela 2.1: Características do Zigbee 35

Tabela 2.2: Potências Variáveis do CC2420 38

Tabela 2.3: Características RSSI 39 


\section{Glossário de Acrônimos}

$\begin{array}{ll}\text { AM } & \text { Active Message } \\ \text { CER } & \text { Chip Error Rate } \\ \text { CRC } & \text { Cyclic Redundancy Check } \\ \text { DTNBOR } & \text { Determine the minimum Transmission power to reach each Neighbor } \\ \text { DTRNG } & \text { Determine transmission power using Relative Neighborhood Graph } \\ \text { FCS } & \text { Frame Check Sequence } \\ \text { IEEE } & \text { Institute of Electrical and Electronics Engineers } \\ \text { LQI } & \text { Link Quality Indication } \\ \text { MST } & \text { Minimum Spanning Tree } \\ \text { PER } & \text { Packet Error Rate } \\ \text { PRR } & \text { Packet Received Rate } \\ \text { RNG } & \text { Relative Neighborhood Graph } \\ \text { RSSF } & \text { Redes de sensores sem fio } \\ \text { RSSI } & \text { Received Signal Strenght Indication } \\ \text { TC } & \text { Topology Control } \\ \text { WSN } & \text { Wireless Sensor Network } \\ \text { WPAN } & \text { Wireless Personal Access Network }\end{array}$

